\title{
Flower pollination optimization based PID controller tuning scheme with robust stabilization of gain scheduled CSTR
}

\author{
T.SUDHA, D. SUBBULEKSHMI \\ School of Electrical and Electronics Engineering, \\ VIT University, Chennai, 600127, Tamil Nadu, INDIA \\ t.sudha2014@vit.ac.in, subbulekshmi.d@vit.ac.in
}

\begin{abstract}
In the area of chemical and control engineering, Continuous Stirred Tank Reactor (CSTR) has been deliberated as a most important topic in temperature process control and it is a extremely nonlinear process, which displays stability only in definite regions and unsteadiness in other regions. In this CSTR, Proportional Integral Derivative (PID) controller has been used to minimize the Integral SquareError (ISE) by adjustment of control parameters like $\boldsymbol{k}_{\boldsymbol{p}}, \boldsymbol{k}_{\boldsymbol{i}}$ and $\boldsymbol{k}_{\boldsymbol{d}}$. But, this conventional controller has high computational complexity, so to reduce this, the optimization based tool has been designed for PID and it was conceptually simple.Earlier, the Genetic Algorithm (GA) optimization has been focused, but it was not guarantee for good fitness and global optimal solution. So to overcome this, here, Flower Pollination based Global Optimization Algorithm (FPGOA) with Gain scheduling based PID controller is proposed for CSTR. When a plant is exposing to big changes in its operational state, the Gain scheduling has been used. The FPGOA is used for tuning the parameters of PID controller for each region to reduce the ISE. The fuzzy gain scheduler has been combined with multiple local linear PID controllers to check the stability of loop for entire regions with various levels of temperatures. This scheduling is an exceptional form of fuzzy control, which customs linguistic rules and fuzzy perceptive to establish the controller factor transition strategy for the dynamic plant dependent on high changes in its operational state to improve the optimization. MATLAB results demonstrate that the feasibility of using the proposed PID controller compared than existing controller, and it shows the FPGOA attained better results, due to the effectual fitness and global optimal solution for the dynamical nonlinear control of CSTR. The response curves of these systems has been generated and compared with existing schemes.
\end{abstract}

keywords: CSTR, PID controller, Flower Pollination, Global Optimization, Gain scheduling, Fuzzy.

Received: June 10, 2019. Revised: December 22, 2019. Accepted: January 13, 2020. Published: January 31, 2020.

\section{Introduction}

PID controllers are still extensively utilized in $90 \%$ of industries, as no other higher control methods like model predictive control, internal model control (IMC), and sliding mode control (SMC) counterpart the effortlessness, obvious functionality, applicability, and easiness of use offered by this controller [1]. PID controller tuned at an exacting operating point will not offer a rewarding response when, there subsists divergence in the method operating series [2]. Therefore, soft computing supported PID controller tuning is extensively anticipated by the researchers throughout the previous few decades [3-8]. CSTR method displays distinctive nonlinear and time varying performance where control of the factor reactor focus with few computation phases is confront to the researchers. Consequently, it becomes necessary to use dominant heuristic algorithms such as GA to tune the PID factors, thus accomplishing highquality set point tracking and disturbance elimination control in the chemical scheme CSTR. Vinodha et al. have suggested three control proposals for nonlinear CSTR method like extraction of PID controller parameters supported on artificial intelligence, model predictive controllers by means of the weighted sum of the output from local predictive controllers, and numerous model PID controllers [9].The existence of bound uncertainty in nonlinear systems such as
CSTR has been forced by designing the Nonlinear Predictive Adaptive Controller (NPAC) and a Nonlinear Disturbance Observer (NDO) in literature [10] and the anticipated recompense technique progresses scheme tracking precision and forcefulness. In the literature, there exists a numeral of techniques to well tune the factor settings of traditional PID controllers. The most well recognized technique is the Ziegler-Nichols (i.e. $\mathrm{ZN}$ ) continuous cycling technique. For an extensive assortment of sensible processes, fine-tuning method facilitates fairly well to fabricate sufficient traditional PID controllers settings. But existing tuning techniques will capitulate PI or PID factors for merely a constrained class of processed models. There is refusal of common method for arbitrary method models similar to them with a first-order / second-order time-delayed replica and applies a suitable rule [11]. As procedure difficulty amplifies this method is unbalanced, the numeral of appropriate tuning rules diminishes and in numerous cases disappears in total. The rules obtainable from certain processes be inclined and ad hoc in nature and not grounded on recital or forcefulness criterion. Optimization is a preferred tool for the controllers. The technique is theoretically easy and is well suitable for PID controllers in which the controller arrangement and parameterization usually specified. There are, nonetheless, numerous pitfalls when by means of optimization. Concern must be 
implemented while formulating criterion and restrictions, or else, a principle will certainly be finest, but controller may be inappropriate as of a deserted constraint. Further intricacy is that the thrashing function may have numerous local minima. In addition, the calculations necessary may be extreme. In general, optimization is a highquality tool which has effectively been utilized to intend PID controllers [12]. This effort, the Flower Pollination supported Global Optimization Algorithm (FPGOA) is utilized for tuning controller factors by reducing integral square error (ISE). The foremost benefit by means of FPGOA that locates global minima. A tuning PID controller has least ISE is encountered to have specified the finest recital [13]. Forcefulness of the controller and engaged in order to create a reasonable comparison.

A gain scheduled control system is examined based on the non-linearity. A gain scheduled control method comprises of a relational controllers (local controllers) and a scheduler. Based on the operating area, the scheduler decides the controller. An overabundance of superior control methods like neural adaptive controller [14], nonlinear internal model control system [15] and fuzzy model predictive control method [16], adaptive control [17], and forceful method control [18] have been functional to the CSTR method measured for the imitation effort in this work. With these nonlinear control schemes, gain scheduling provides easiest and least computational complication.

Gain scheduling is a renowned method for industrial control $[19,20]$ and utilized when the plant focus on great transformations in the operating state, a circumstances that is characteristic in industry. Huge transformation in the operating state directs to consequent variations in the factors of the linearized replicas of the plant concerning these operating states; it is organized and not probable to invent a controller to activate acceptably one operating state and anticipate to execute uniformly well elsewhere devoid of retuning it. Closed system recital is corrupted as the controller was not attempted tomodify the operating states. Substantial work has departed the controller's development that can trail the plant factors variationswith a point to accomplish invariant operation all through the operational area of the plant. Adaptive controller is a technique that devoid of constantly reveal suitable recital all through the operational region of the plant, on this view, misplace control in total. Robust controller, subsequent technique, also has its restrictions as it must pact with dynamicsystem that diverge over extensive choice whilst by means of merely constant factors. Obviously this controller class can function merely over a partial field. Fuzzy gain scheduling guarantees smoother transitions among direct law that sustains fundamentally invariant closed scheme.
In this research, online tuning is carried out for PID factors for the diverse areas independently by FPGOA. The tuned PID factors are fuzzy gain programmed to offer the suitable values for diverse areas. Simulation outcomes demonstrate the probability by means of the anticipated controller to manage the dynamical nonlinear CSTR. Association of the work is offered as trails. Section 2 provides the literature survey of CSTR method supported PID factor tuning techniques. A section 4 offer anticipated control method and depicts the CSTR plant. Beginning to FPGOA is given in Section 4 and Section 5 comprises the simulation outcomes of CSTR method for both servo and regulatory cases. At last, Section 6 end ups how FPGOA supported PID controller offers improved performance with least computation phases to accomplish optimized PID control of the CSTR plant in an extensive range.

\section{Related Work}

Nagaraj and Murugananth have examined PID tuning by means of soft computing grounded controller, enhancing the recital of method in terms of time domain stipulations, set point tracking, and regulatory modification [21]. In the study of Chang, PID controller has been intended by means of an artificial bee colony procedure for an unstable and nonlinear CSTR chemical scheme [22]. Wang and Jin have described state feedback linearization control technique for non-minimum phase features of CSTR scheme [23]. Singh and Sharma have anticipated a fractional order PID controller with a Particle Swarm Optimization (PSO) method, offering enhanced servo and regulatory response [24]. The merged PID control tuning by coupling the gain-phase margin technique with genetic algorithm has been functional for position control in a servo scheme [25]. A virtual feedback control has been executed to manage the state variables by means of Extended Kalman filter (EKF) in the feedback path via particle swarm optimization method for CSTR method [6].

Jayachitra \& Vinodha [8] proposed Genetic algorithm (GA) with respect to PID (proportional integral derivative) controller. The controller produced optimized PID tuning factors in a continuous stirred tank reactor (CSTR) method. The weighted objective functions, especially, integral absolute error (IAE), integrated time absolute error (ITAE) and integral square error (ISE). The primary goal of biochemical and chemical industries is the optimization of PID controller. With forceful nonlinearity PID controllers tape down the operating range. To overcome the restrictions of linear PID controller, global optimized PID factors activates the CSTR method in the proposed method. The GA based PID controller along with the fixed PID 
factors shows satisfactory performance in the trailing point and disturbance elimination.

NAGARAJU et al., [26] designed a controller is intended for controlling the deliberation of one chemical with the assistance of other. This work features the manipulation of diverse controllers such as PI, PID, Fuzzy logic controller and GA ahead the method model. Model design and simulation are performed in the MATLAB/ SIMULINK software. The deliberation control is found improved controlled with the accumulation of GA as a substitute of fuzzy logic and predictable PID controllers. The enhancement of the method is observed. Jau-Woei Perng et al., [27] demonstrates the recognition of optimal controller gain for the Fractional Order Proportional Integral Derivative (FOPID) controller based on the Stochastic Inertia Weight Particle Swarm Optimization (SIWPSO) algorithm andRadial Basis Function Neural Network (RBFNN) techniques. The 3D stability regions such as $\boldsymbol{k}_{\boldsymbol{p}}, \boldsymbol{k}_{\boldsymbol{i}}$ and $\boldsymbol{k}_{\boldsymbol{d}}$ factor space are given in the grsaphic representation. This experimentation offers an intelligent SIWPSO-RBF procedure for recognizing the optimal result for a FOPID control scheme. To elucidate the use of SIWPSO-RBFNN technique, this workprovides twocases elucidating the anticipated algorithm that can be functional with two fractional-order time-delayschemes and FOPIDtype controllers. Moreover, the anticipated algorithm can be utilized in two required methods if transfer functions of the systems are identified. The initial process engages recognizing the optimal $\boldsymbol{k}_{\boldsymbol{p}}$ and $\boldsymbol{k}_{\boldsymbol{i}}$ gains while $\boldsymbol{k}_{\boldsymbol{d}}$ vary and the factors $\lambda$ and $\mu$ are identified. The subsequent method entails recognizing the optimal $\boldsymbol{k}_{\boldsymbol{p}}$, gains whilst $\lambda$ and $\mu$ vary. Ultimately, numerous limitations of the anticipated algorithm demonstrated the efficiency of a FOPID controller concerning fractional-order with time-delay schemes.

Rahmatet al., [28] offered two diverse control schemes supported on the amalgamation of a new socio-political optimization algorithm, termed Imperialist Competitive Algorithm (ICA), and idea of the gain scheduling executed using the least square and fuzzy logic methods. The aim is to manage the temperature of theCSTR in existence of the set point changes. The works trailed with scheming those controllers and imitating in MATLAB software. The recital of the proposed controllers have been consider supported on the Sum of the Square Error (SSE) and Integral Absolute Error (IAE) measure. The outcomes noticeably point out that mutually recommended control approaches present an acceptable performance relating to the functional modifications of the method. In other word, forcefulness of the anticipated methods in dealing uncertainties all over the tracking of the reference signal take the spotlights these point into account. In addition, fuzzy based structure policy offers the highly flexibility and specific behaviour in control action in contrast to the least square based method.

\section{Process Description}

Reactors participate in a primary role in several chemical industries. In the CSTR method replica under conversation, an irreversible exothermic reaction happens. Figure 1 illustrates the sketch of the CSTR model.

The heat reaction is partitioned by the coolant medium with respect to the jacket around the reactor. ' $A$ ' (i.e. a fluid stream) is given inside the reactor as well as a compound is located inside the reactor. The out valve is used to send the fluid out which surrounds the reactor. The jacket adjacent to the reactor also consists of outlet streams and feed. The temperature is lower and supposed to be mixed in the jacket contrast to the reactor. This is a MultiInput, Multi-Output (MIMO) method. It is categorized by differential formulas through energy and mass balances.

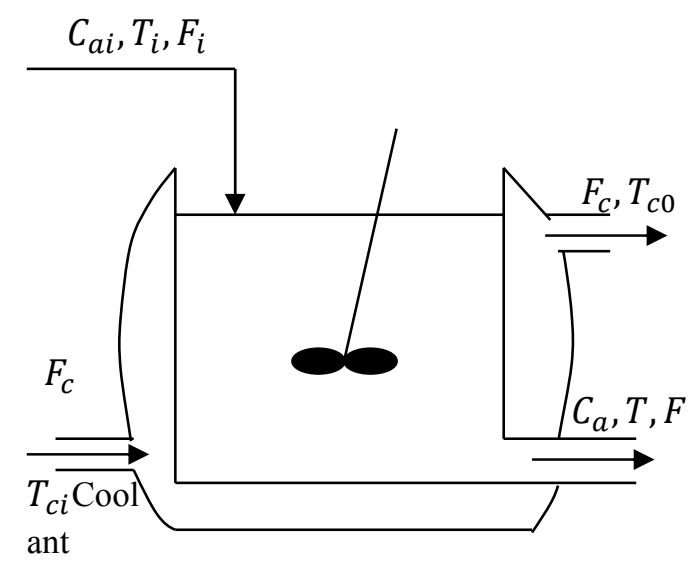

Figure 1: CSTR process diagram

The reactor model placed in a material and energy balance formulas is specified by

$$
\begin{gathered}
\frac{d T}{d t}=\frac{F_{i}}{V}\left(T_{i}-T\right)+\frac{U_{A}}{V_{\rho} C_{p}}\left(T_{c}-T\right) \\
-\frac{k_{o} \Delta H}{\rho C_{p}} e^{-E / R T} C \\
\frac{d C}{d t}=\frac{F_{i}}{V}\left(C_{i}-C\right) k_{o} e^{-E / R T} C \\
\frac{d T_{c}}{d t}=F_{c}\left(T_{c i}-T_{c}\right)+\frac{U_{A}}{C_{p c}}\left(T_{c}-T\right)
\end{gathered}
$$

Where $\mathrm{T}=$ the temperatue and $\mathrm{C}=$ concentration of the reactor correspondingly, $\boldsymbol{F}_{\boldsymbol{i}}=$ input flow and $\boldsymbol{T}_{\boldsymbol{i}}=$ reactant temperature, $\mathrm{V}=$ the flowing rate of the inlet reactants volume with respect to CSTR method, and $\mathrm{Fc}$ is coolant flow and $\boldsymbol{T}_{\boldsymbol{c} i}=$ temperature. The significant outputs are $\mathrm{C}$ which is 
concentration, $\mathrm{T}=$ product temperature, and $\boldsymbol{T}_{\boldsymbol{c}}$, $=$ coolant temperature. CSTR parameters are shown in given below Table I.

Table 1: Parameter values of CSTR

\begin{tabular}{|c|c|}
\hline Parameters & values \\
\hline $\boldsymbol{U}_{\boldsymbol{A}}, \mathrm{m}^{2}$ & 0.5 \\
\hline $\boldsymbol{F}_{\boldsymbol{i}}, \mathrm{m}$ & 2 \\
\hline $\boldsymbol{k}_{\boldsymbol{o}}, \mathbf{h}^{-\mathbf{1}}$ & $34.9308 \times 10^{6}$ \\
\hline$-(\Delta \boldsymbol{H}), \mathrm{kcal} / \mathrm{kgmol}$ & 11.92 \\
\hline $\boldsymbol{E} / \boldsymbol{R} \boldsymbol{T}, \boldsymbol{C}$ & 5963.6 \\
\hline $\boldsymbol{T}_{\boldsymbol{i}}, \boldsymbol{K}$ & 295 \\
\hline $\boldsymbol{C}_{\boldsymbol{i}}, \boldsymbol{k g} \boldsymbol{m o l} / \boldsymbol{m}^{\mathbf{3}}$ & 2 \\
\hline $\mathrm{V}, \mathrm{kcal} /\left(\mathrm{m}^{2} \mathrm{~K} \mathrm{~h}\right)$ & 0.6 \\
\hline $\boldsymbol{T}_{\boldsymbol{c}}, \boldsymbol{K}$ & 295 \\
\hline
\end{tabular}

On investigation, the equations of nonlinear terms are given as $\boldsymbol{e}^{-\boldsymbol{E} / \boldsymbol{R} \boldsymbol{T}} \boldsymbol{C}$ as shown in Eqns (1) and (2) also $\boldsymbol{F}_{\boldsymbol{c}} \boldsymbol{T}_{\boldsymbol{c}}$ and $\boldsymbol{F}_{\boldsymbol{c}} \boldsymbol{T}_{\boldsymbol{c} \boldsymbol{i}}$ in Eqn (3). Linearization of the non-linear phase is performed in the $\left(\boldsymbol{C}_{\mathbf{0}}, \boldsymbol{T}_{\mathbf{0}}\right)$ operating point. Taylor's series and linearization are related based on the nonlinear term $\boldsymbol{e}^{-\boldsymbol{E} / \boldsymbol{R} \boldsymbol{T}} \boldsymbol{C}$,

$$
\begin{array}{r}
e^{-\Delta E / R T_{0}} C_{0}+\frac{E}{R T_{0}^{2}} e^{\frac{-\Delta E}{R T_{0}}} C_{0}\left(T-T_{0}\right) \\
+e^{-\frac{\Delta E}{R T_{0}}}\left(C-C_{0}\right)
\end{array}
$$

By applying linearized formula, Eqns (1)-(3) is translated into deviation variable outline:

$$
\begin{aligned}
& \frac{d C^{\prime}}{d t}=\left[\frac{F_{i}}{V}\left(C_{i}-C\right) k_{o} e^{-\frac{E}{R T_{0}}}\right] C^{\prime}-k_{0} \frac{E}{R T_{0}^{2}} e^{\frac{-\Delta E}{R T_{0}}} C_{0} T^{\prime}+\frac{F_{i}}{V} C_{i}^{\prime} \\
& \frac{d T}{d t}=J k_{0} \frac{E}{R T_{0}^{2}} e^{\frac{-\Delta E}{R T_{0}} C^{\prime}} \\
&+\left[J k_{0} \frac{E}{R T_{0}^{2}} e^{\frac{-\Delta E}{R T_{0}}} C_{0}-\frac{U_{A}}{V_{\rho} C_{p}}\right. \\
&\left.-\frac{F_{i}}{V}\right] T^{\prime}+\frac{U_{A}}{V_{\rho} C_{p}} T_{c}^{\prime}+\frac{F_{i}}{V} T_{i}^{\prime} \\
& \frac{d T^{\prime}{ }_{c}}{d t}=F_{c}^{\prime}{ }_{c}\left(T_{c i}-\right.\left.T_{c 0}\right)+\left[\frac{U_{A}}{\rho C_{p c}}-F_{c 0}\right] T_{c}^{\prime} \\
&-\frac{U_{A}}{\rho C_{p c}} T^{\prime}
\end{aligned}
$$

The typical feed concentration of $\mathrm{A}$ is given by 10 $\mathrm{kgmol}=\mathrm{m} 3$ and the reactor concentration of $\mathrm{A}$ is given as a series $0<\mathrm{CA}<10$. The lower bound temperature is given as $298 \mathrm{~K}$, when there is no reaction, whilst the feed and jacket temperature are $298 \mathrm{~K}$. The concentration and temperature is shown as correlation co-efficient. Slight reaction is shown when the awareness of A is huge, hence slight energy is projected in case of reaction, and also temperature will not vary greatly as that of feed and jacket temperatures.

The stability characteristics of the eigen values $\mathrm{A}$, which are attained by working out $|\lambda \boldsymbol{I}-\boldsymbol{A}|$.

$$
|\lambda I-A|=\left[\begin{array}{ccc}
\lambda-a_{11} & -a_{12} & -a_{13} \\
-a_{21} & \lambda-a_{22} & -a_{23} \\
-a_{31} & -a_{32} & \lambda-a_{33}
\end{array}\right]
$$

Piecewise linearization is performed when $\mathrm{I}=0$ as shown in figure 2. In all the regions state space matrices must be set up to pertain linear control methods, thus the nonlinear plants are partitioned into three linear regions.

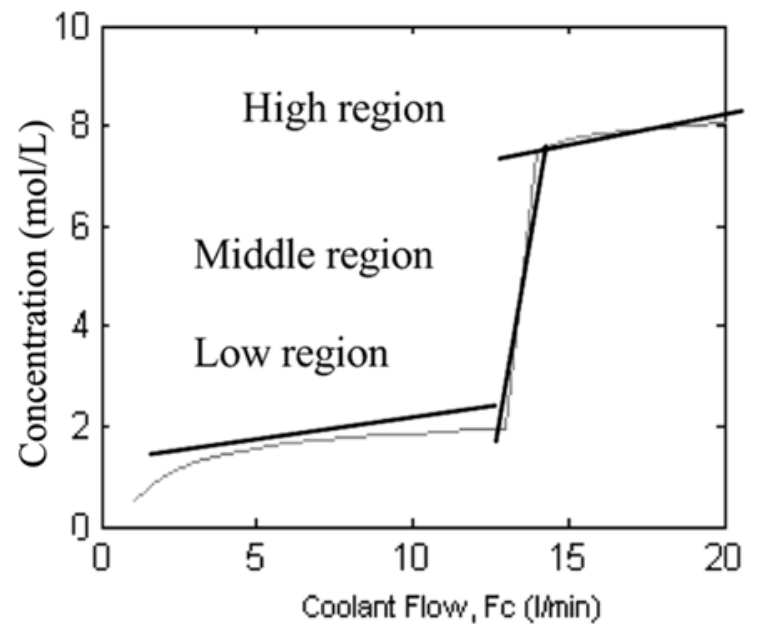

Figure 2: Three linear regions based Inputoutput characteristics

The operating region and Eigen values for the diverse areas are displayed in Table II. On examining the Eigen values, it is obvious that the

\begin{tabular}{|c|c|c|c|c|c|}
\hline Region & $F_{c 0}$ & $C_{a 0}$ & $T_{0}$ & $T_{c 0}$ & Eigenvalues \\
\hline $\begin{array}{l}\text { Low } \\
\text { region }\end{array}$ & 2 & 5.5 & 380 & 318 & $\begin{array}{l}-5.3375 \\
-2.0010 \\
-3.9091\end{array}$ \\
\hline $\begin{array}{l}\text { Middle } \\
\text { region }\end{array}$ & 8 & 7.5 & 339 & 305 & $\begin{array}{l}7.1998 \\
-7.9998 \\
-5.3305 \\
\end{array}$ \\
\hline $\begin{array}{l}\text { High } \\
\text { region }\end{array}$ & 16 & 11.5 & 320 & 302 & $\begin{array}{l}-0.2816 \\
-16.0000 \\
-8.1735\end{array}$ \\
\hline
\end{tabular}
positive Eigen value is relating to middle area, stating instability conversely; that is, the middle area provides open-loop unstablility. On the contrary, the Eigen values are negative in the low/high areas, identifying them to be open-loop stable. Therefore, control of certain complicated method is hard to execute.

Table 2: Eigen values at various operating conditions 


\section{Flower pollination algorithm for global optimization}

Transmitting the pollens from flowers is associated with the Flower pollination. The primary actors involved in performing transfers are bats, insects, birds and other animals. A flower-pollinator partnership is engaged by several insects and flowers as described. The insects are the principal pollinators and the partnership is made only between the flowers and the associated birds as proposed by [29].

There exists two sorts of pollination: biotic and abiotic. In which Biotic engages $90 \%$ of flower pollination, whilst abiotic inhabits $10 \%$. Abiotic pollination requires no pollinators. Several insects be inclined to definite kinds of flowers; at the similar time, the insects circumvent other flower species, and this occurrence is termed: flower constancy [30, 31]. All flowers possess flower constancy possessions and have assurance of reproduction maximization. Self-pollination and cross-pollination paved the way for flower pollination method. The pollens stared relocating to different plants in case of cross-pollination whereas the insects such as bees, birds and bats fly for long distances for the happening of cross pollination. The above mentioned flies are considered as the global pollinators. Bees and birds typically follow the characteristics of Levy flight [32]; this shows that the discrete moves cause the significance of levy distributions. The self- pollination is a subsequent pollination which follows fertilization.Fertilization process is accounted in the self-pollination based on the similar kind of flowers. Self-pollination usuallynecessitates no pollinators.

The features relating the flower constancy, pollination method and behaviour of pollinators given way to the following rules:

- (Rule 1): The cross-pollination and thebiotic is given as a global pollination, while the pollinators associates with the Levy distribution.

- (Rule 2): A local pollination can be given as the abiotic and self-pollination.

- (Rule 3): Reproduction fraction propotenional to degree of relationship between two diverse flowers and it can be measured as the flower constancy.

- (Rule 4): Local pollination has superior benefits than the global pollination with respect to the physical proximity and wind. The value of variable $P$ ranges between $[0$, $1]$.

The fittest reproduction is provided based on the movement of the insects to the long distance, with regards to global pollination. The mathematical origination of flower constancy and primary rule is shown when the fittest is provided as $\mathrm{g}^{*}$.

$x_{i}^{t+1}=x_{i}^{t}+\gamma L\left(g^{*}-x_{i}^{t}\right)$

In Eq. (1), $\boldsymbol{x}_{\boldsymbol{i}}^{t}$ signifies I pollen; in subsequent words, $\boldsymbol{x}_{\boldsymbol{i}}^{\boldsymbol{t}}$ is a result vector at iteration $\mathrm{t}, \boldsymbol{g}^{*}$ is the finest obtained solution at iteration $\mathrm{t}, \gamma$ signifies the scaling factor of step size, and L is the pollination potency or step size. The insect's extensive shift can mimicked by Levy flight [29]. For this reason, the Levy distribution derives the step size L.

The flower constance and local pollination is originated as given in rule 2 :

$$
x_{i}^{t+1}=x_{i}^{t}+\epsilon\left(x_{j}^{t}-x_{k}^{t}\right)
$$

Where $\boldsymbol{x}_{\boldsymbol{j}}^{\boldsymbol{t}}$ and $\boldsymbol{x}_{\boldsymbol{k}}^{\boldsymbol{t}}$ are pollens (solution vectors) in which transmitted from diverse flowers, but those flowers are located in a sole plant species. This sort of flower constancy has small neighbourhood. The variable is copied from the range of $[0,1]$ in an uniform distribution. The pollination method possesses either local or global, as switch probability $\mathrm{P}$ is offered to transform among the two pollination types (Rule 4).

\section{Proposed Online FPGOA-Based PID Tuning Minimizing Integral Square Error (ISE)}

In the literature, unbalanced Single Input and Multiple Output (SIMO) method has not been forced in accordance to the Tuning Conventional PID factors. By reducing ISE, GA is cast off for an auto-tuning PID controllers for linear stable and unstable regions of individual. As given below, PID controller offers non interacting form and it is essential to compute minimum set of attributes based on the PID law of permutations for control stratergy. By appropriate transformation of the factors this type is transformed to the interrelated outline. Reducing the subsequent error principle produces the controller factor. FPGOA is cast off as an auto-tuning PID controllers for entity linear unstable and stable areas by diminishing ISE. Owing to the diversity of PID permutations control law, it is essential to identify a least attribute set, in which, the PID controller is treated as a noninteractingtype as distinct below:

$$
G_{c}(s)=K_{p}+K_{i} \frac{1}{s}+K_{d} s
$$

By suitable transformation factor, the structure is moved to an interacting form. Reduce the subsequent error measure operates the controller factor

$$
I S E=\int_{0}^{T}[r(t)-y(t)]^{2} d t
$$


Where $\boldsymbol{r}(\boldsymbol{t})=$ referenceinput, and $\boldsymbol{Y}(\boldsymbol{t})=$ measured variable.

Where $\boldsymbol{r}(\boldsymbol{t})=$ referenceinput, and $\boldsymbol{Y}(\boldsymbol{t})=$ measured variable.

In this investigation, the measurement of error is minimizedbased on the genetic algorithm. Figure 4 illustrates the schematic representation for PID factors online tuning by means of FPGOA while decreasing ISE. Simulation outcomes are provided for three diverse operating regions.

When the population size is 0 , the population category is fixed as double vector. The standardized creation function is selected when the range falls between $0-100$.

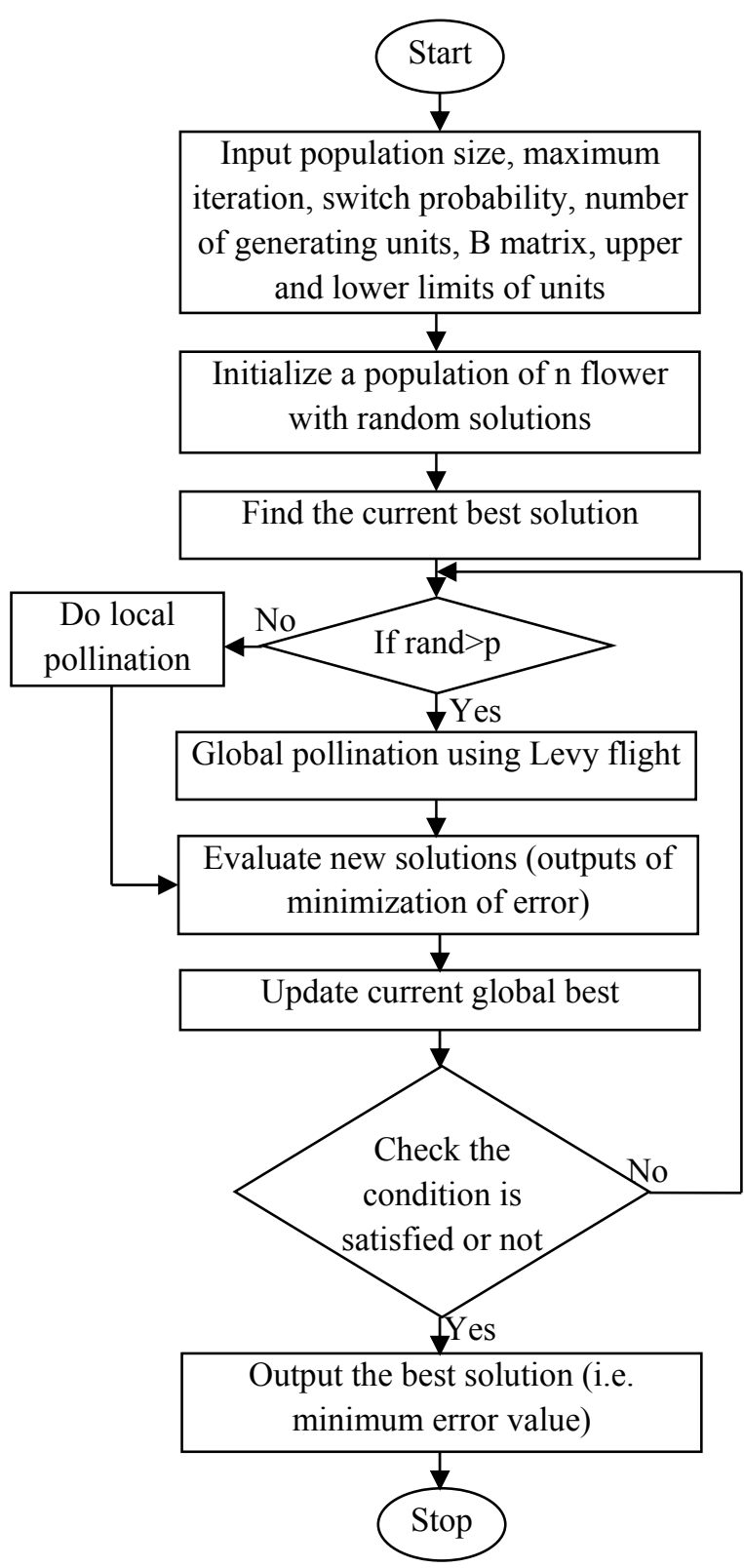

Figure 3: FPGOA based PID parameter tuning flow chart

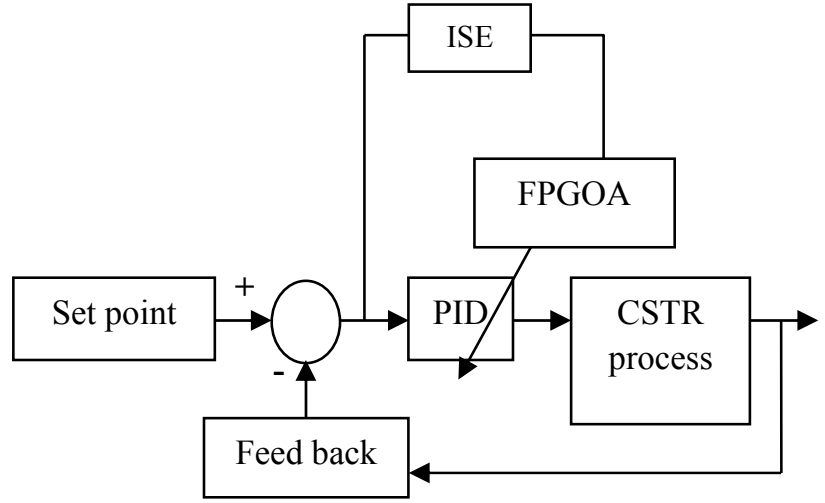

Figure 4: Parameters of online PID tuning using FPGOA based block diagram

Algorithm 1: FPGOA based ISE minimization
procedure

Input: initialize the $\mathrm{n}=50$, switch probability $\mathrm{P}=$ $0.8, \gamma=0.01$, for Levy flight $\lambda=1.5$

ISE error criterion is minimized the objective function is mentioned in equation (14)

an random initial population has been created and pop size $n$

the best solution in pop of $\mathbf{g}^{*}$ has been identified

$\mathbf{P} \in[\mathbf{0}, \mathbf{1}]$ identified, which is a switch probability between global and local pollination

While (gen $<$ max generation num)

For $\mathrm{i}=1$ to $\mathrm{n}$

If rand $>\mathrm{p}$ then

the vector $\mathrm{L}$ has been Drawn from the levy distribution $\lambda$, $\mathrm{L}$ has $\mathrm{d}$ dimension

the global pollination has been applied via $\mathbf{X}_{\mathbf{i}}^{\mathbf{t + 1}}=$ $\mathbf{X}_{\mathrm{i}}^{\mathrm{t}}+\gamma \mathbf{L}\left(\mathbf{g}^{*}-\mathbf{X}_{\mathbf{i}}^{\mathrm{t}+\mathbf{1}}\right)$

Else

Illustrate $\boldsymbol{\epsilon}$ as of from a uniform distribution in $[0,1]$

$\mathrm{j}$ and $\mathrm{k}$ randomly chosen amongst the solutions

Do local pollination via $\mathbf{X}_{\mathbf{i}}^{\mathbf{t + 1}}=\mathbf{X}_{\mathbf{i}}^{\mathbf{t}}+\boldsymbol{\epsilon}\left(\mathbf{X}_{\mathbf{j}}^{\mathbf{t}}-\mathbf{X}_{\mathbf{k}}^{\mathbf{t}}\right)$

End if

the fitness of the new solution has been calculated

If fitness of the new solution is superior than the old one, then replace it

End for

Determine the current best solution $\mathbf{g}^{*}$

$$
- \text { gen }=\text { gen }+1
$$

End while 


\section{Sugeno fuzzy model based Gain Scheduler for CSTR process}

Usually, the PID factors tuned for higher area yield and reduced ISE for low area. The unstable closedloop response was obtained when the factors associated with the higher area is utilized for middle area. To activate CSTR for the entire range, merely gain scheduling was necessary. The schematic representation of the fuzzy gain scheduled based PID-controlled CSTR is illustrated in Figure 5. The partitioning of this method is done in three regions: high, middle and low. With the CSTR'S subsequent regions, the GA performance are done primarily by the PID factors. Gain scheduling has been performed to control the entire process.

Since the functionality of the exogenous variable has overflow concentration, the factors (i.e., gains) of the PID controllers are different in a gain scheduling controllers. The factors of the local linear PID factors merged with the Sugeno fuzzy performance to deliver gain scheduling of PID factors. The performance of Sugeno fuzzy is generated with the attentiveness of the fuzzy model input and with the output of $\mathrm{kp}, \mathrm{kd}$ and ki. The membership function input is trapezoidal.

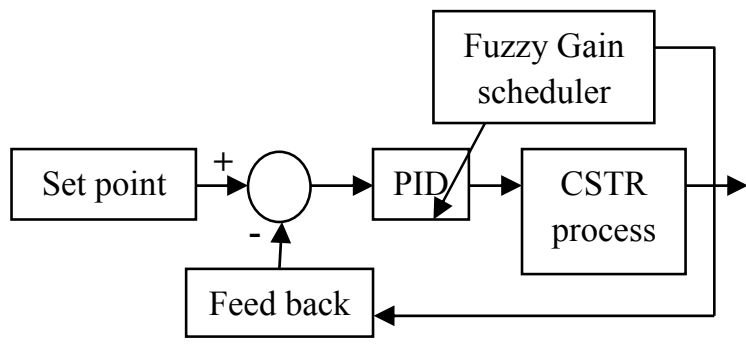

Figure 5: Fuzzy gain scheduled for CSTR based block diagram

\section{Results and Discussion}

This block shows that the recommended FPGOA based PID controller factors in association with tuning performance arematch up to with prevailing Genetic Algorithm [25] based PID controller.

Table 3 offers presentation index for the PID controller factors at diverse operating areas. Figure 6 exposes the FPGOA training for the greater region for least ISE. Figures 7 demonstrate the closed-loop reaction of the CSTR which functions in the diverse regions independentlyby means of the FPGOAtuned PID factors.

Table 3: PID controller factorsstandards at diverse operating regions

\begin{tabular}{|c|c|c|c|c|}
\hline Region & $\boldsymbol{k}_{\boldsymbol{p}}$ & $\boldsymbol{k}_{\boldsymbol{i}}$ & $\boldsymbol{k}_{\boldsymbol{d}}$ & ISE \\
\hline $\begin{array}{c}\text { High } \\
\text { region }\end{array}$ & 9.5624 & 7.0873 & 3.4522 & 6.60 \\
\hline
\end{tabular}

\begin{tabular}{|c|c|c|c|c|}
\hline $\begin{array}{c}\text { Middle } \\
\text { region }\end{array}$ & 93.654 & 18.945 & 57.759 & 88.52 \\
\hline Low region & 7.5125 & 9.475 & 4.3141 & 1.017 \\
\hline
\end{tabular}

Figure 6 exposes the FPGOA training for the greater region for least ISE. In this, 6(a) and (b) shows the graphical representation of low region based concentration and temperature of existing GA and proposed FPGOA schemes. Figures 7 demonstrate the closed-loop reaction of the CSTR functioned in the diverse regions independently by means of the FPGOA-tuned PID factors. Figure 7(a) and 7(b) shows the graphical representation of medium region based concentration and temperature of existing and proposed schemes. Figure 8(a) and 8(b) shows the graphical representation of high region based concentration and temperature of existing and proposed schemes. All the figures are illustrates, the proposed scheme attained less concentration and temperature compared than existing GA, due to the global optimum behaviour. It identifies less ISE, so the results attained standard manner.

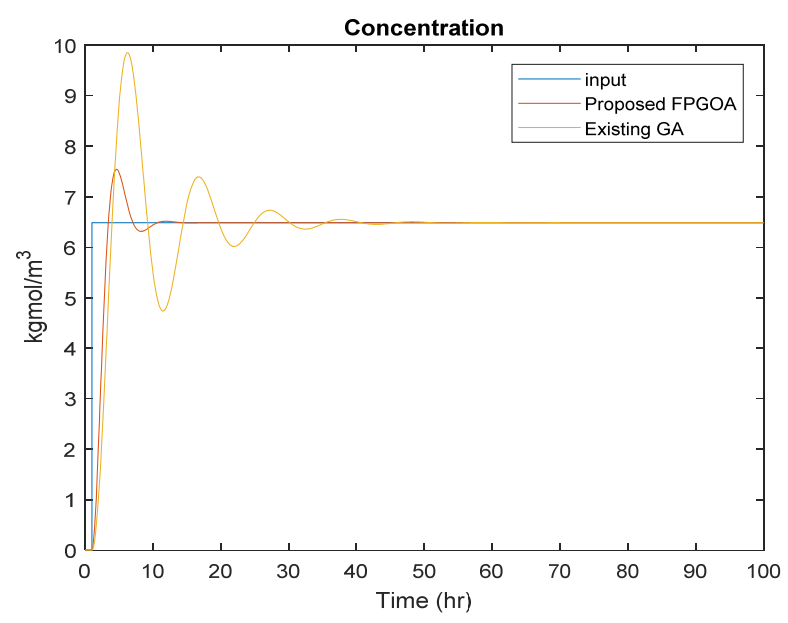

Figure 6(a): low region based concentration performance comparison

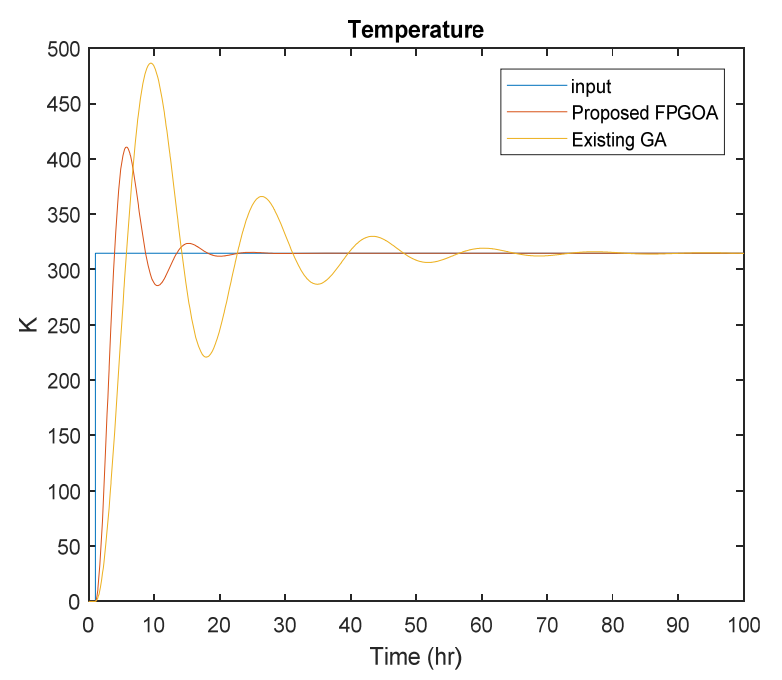


Figure 6(b): low region based temperature performance comparison

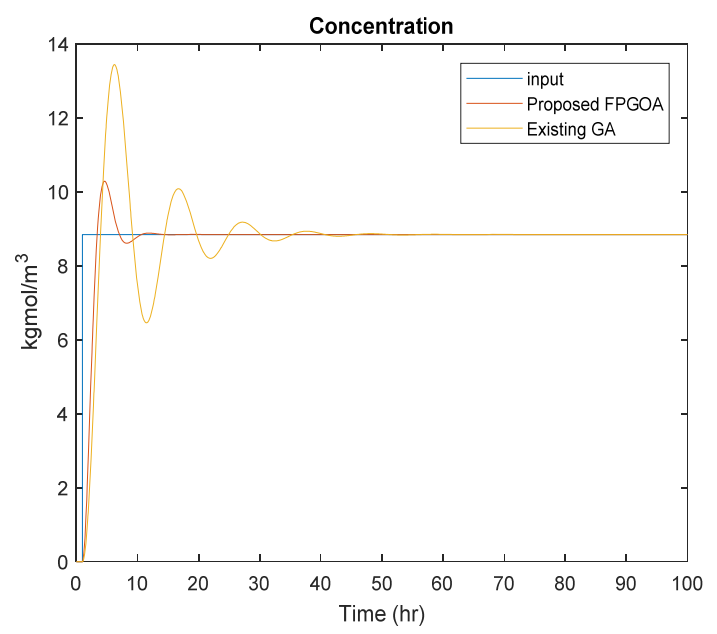

Figure 7(a): medium region based concentration performance comparison

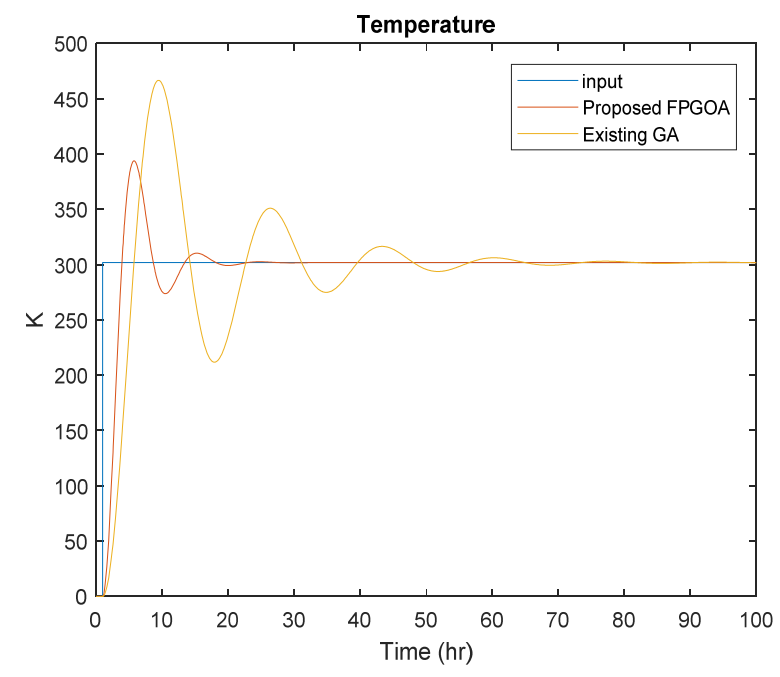

Figure 7(b): medium region based temperature performance comparison

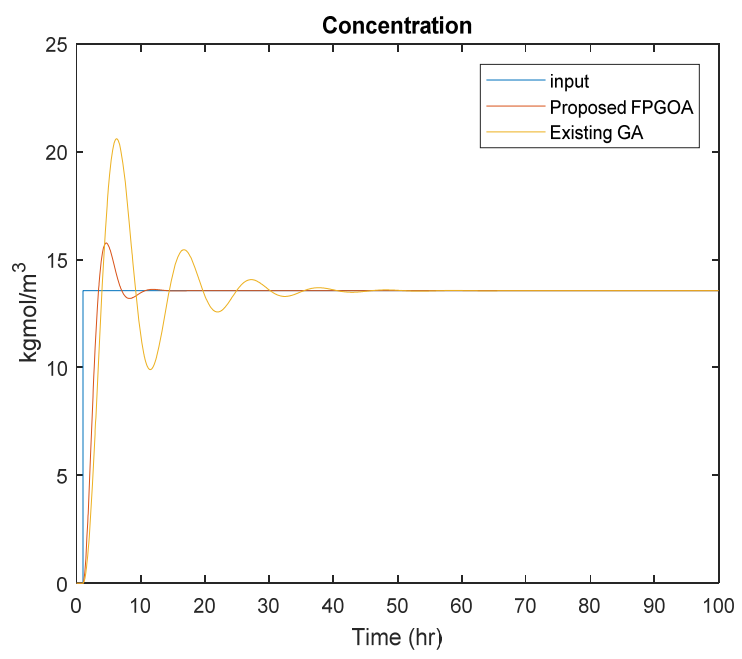

Figure 8(a): high region based concentration performance comparison

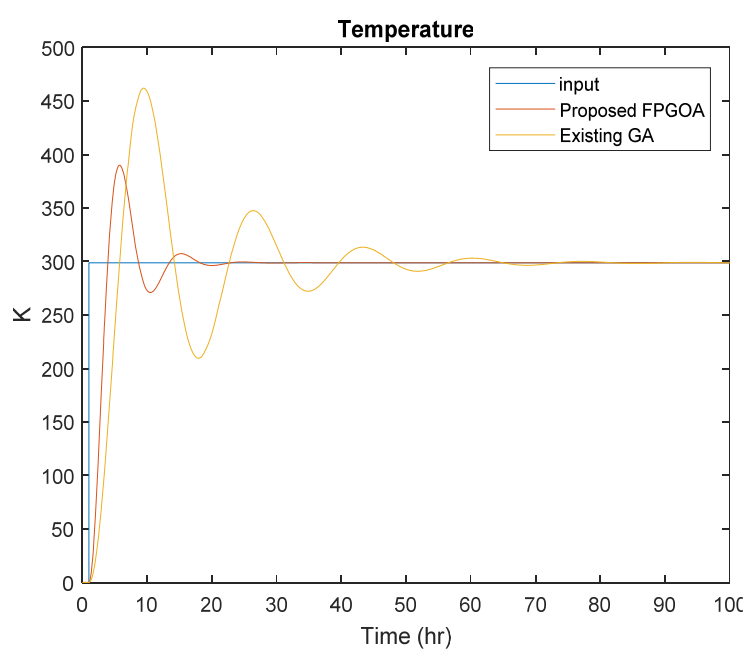

Figure 8 (b): high region based temperature performance comparison

From the above observation the steady state condition for all the three fuzzy states are obtained quickly with the optimized PID values stated in the table 3 . The obtained figures 6,7 and 8 shows that perform of proposed system gives better settling point compared to the existingsystem.

\section{Conclusion}

In research, CSTR method which contributes greater nonlinear features, corresponds to the gain scheduled FPGOA-sourced PID Controller. This feature provides PID controller tuning with the guidance FPGOA. Thus, the Integral Square Error (ISE) has been reduced which spotlights on the stable low or high areas and an unstable middle area. The integration of enormous local linear PID controllers are done through Sugeno Fuzzy (SF) gain scheduler. The main intention of the proposed controller is to reduce ISE and to offer better performance mutually in both set-point trailing and regulatory circumstances.

\section{References}

[1] K. Astrom and T. Hagglund, PID Controllers: Theory, Design, and Tuning, Instrument Society of America, Research Triangle Park, NC, USA, 1994.

[2] A. O. Dwyer, Handbook of PI and PID Controller Tuning Rules, Imperial College Press, 3rd edition, 2009.

[3] Kesarkar, A. A., \& Selvaganesan, N, “Tuning of optimal fractional-order PID controller using an artificial bee colony algorithm," Systems Science \& Control Engineering, Vol.3, Issue.1, Pp. 99-105, 2015. 
[4] A. Karimi, H. Eskandari, M. Sedighizadeh, A. Rezazadeh, and A. Pirayesh, "Optimal PID controller design for AVR system using new optimization algorithm", International Journal on Technical and Physical Problems of Engineering, vol. 5, Issue. 15, pp. 123-128, 2013.

[5] V. Rajinikanth and K. Latha, "Controller parameter optimization for nonlinear systems using enhanced bacteria foraging algorithm", Applied Computational Intelligence and Soft Computing, vol. 2012, Article ID 214264, 12 pages, 2012.

[6] Subbulekshmi, D, "Comparison of Kalman and Extended Kalman Filter in Level-Temperature Cascaded Process", International Journal of Chemical Sciences, vol.14, Issue.3, pp.16701682, 2016.

[7] D. Subbuleskshmi, "Design of LQR With Estimator and 2-DOF PID For A CSTR System," International Journal of Applied Engineering Research, India, Vol. 9, Issue. 22, pp. 16353-16361,2014

[8] Jayachitra.A \& Vinodha.R, "Genetic algorithm based PID controller tuning approach for continuous stirred tank reactor", Advances in Artificial Intelligence, Vol.2014, Article Id: 791230, pp. 1-8, 2014.

[9] R. Vinodha, S. A. Lincoln, and J. Prakash, "Design and implementation of simple adaptive control schemes on simulated model of CSTR process," International Journal of Modelling, Identification and Control, vol. 14, Issue. 3, pp. 159-169, 2011.

[10]H. Man and C. Shao, "Nonlinear predictive adaptive controller for CSTR process," Journal of Computational Information Systems, vol. 8, Issue. 22, pp. 9473-9479, 2012.

[11] Rajinikanth. V \& Latha. K, "Setpoint weighted PID controller tuning for unstable system using heuristic algorithm", Archives of Control Sciences, Vol.22, Issue .4, pp. 481-505, 2012.

[12] Astrom, K. J. and Hagglund, T. "Automatic tuning of PID controllers", in: The Control Handbook, IEEE Press, New York, pp.198-209, 1996.

[13] Jaen-Cuellar, A. Y., de J. Romero-Troncoso, R., Morales-Velazquez, L., \& Osornio-Rios, R. A. "PID-controller tuning optimization with genetic algorithms in servo systems" International Journal of Advanced Robotic Systems, Vol.10, Issue.9, pp. 324, 2013.

[14] Krishnapura, V. G. and Jutan, A, "A neural adaptive controller", Chemical Engineering Science, Vol. 55, pp.3803-3812, 2000.
[15] Nahas, E. P., Henson, M. A., and Seborg, D. E. "Nonlinear internal model control strategy for neural network models", Computers and Chemical Engineering, Vol. 16, Issue.12, pp. 1039-1057,1992.

[16]Huang, Y. L., Lou, H. H., Gong, J. P., and Edgar, T. F, "Fuzzy model predictive control", IEEE Transactions on Fuzzy Systems, Vol.8, Issue 6, pp.665-678, 2000.

[17] Astrom K. J and Wittenmark, B, "Adaptive Control", Pearson Education Press, Boston. 2001.

[18] Morari, M., \& Zafiriou, E. "Robust process control Englewood Cliffs", NJ: Prentice hall. pp. 116-117, 1989.

[19]Zhao, Z.Y, Tomizuka, M, and Isaka, S, "Fuzzy gain scheduling of PID controllers", IEEE Trans. Syst. Man Cybern, Vol.23, Issue.5, pp. 1392-1398, 1993.

[20] Jaya.N, Sivakumar, D, and Anandanatarajan.R, "Implementation of fuzzy gain scheduling of PID controllers for a two capacity interacting nonlinear process", paper presented at TIMA, January 2007.

[21] Ouyang, P., \&Pano, V,“Comparative study of DE, PSO and GA for position domain PID controller tuning. Algorithms", Vol.8, Issue.3, pp.697-711, 2015.

[22] W.-D. Chang, "Nonlinear CSTR control system design using anartificial bee colony algorithm", Simulation Modelling Practice and Theory, vol. 31, pp. 1-9, 2013.

[23] W. Wang and X. Jin, "An optimization tuning method of nonlinear non-minimum phase systems and its application to chemical process", in Proceedings of the 26th Chinese Control and Decision Conference (CCDC '14), pp. 4929-4935, Changsha, China, June 2014.

[24] Chaturvedi, S., \& Kumar, N, "Comparative Analysis of CSTR Concentration Control Using Different Soft Computing Techniques", International Journal of Applied Engineering Research, Vol.9, Issue.11, pp. 1337-1346, 2014.

[25] A. Y. Jaen-Cuellar, R. D. J. Romero-Troncoso, L. Morales-Velazquez, and R. A. Osornio-Rios, "PID-controller tuning optimization with genetic algorithms in servo systems," International Journal of Advanced Robotic Systems, vol. 10, pp. 324, 2013.

[26] Nagaraju, D. Siva, and G. Ramesh. "Design and implementation of intelligent controllers for a continuous stirred tank reactor system", International Journal for Research in Applied Science \& Engineering Technology 
(IJRASET), Volume 2, Issue XI, November 2014

[27] Perng, Jau-Woei, Guan-Yan Chen, and Ya-Wen Hsu. "FOPID controller optimization based on SIWPSO-RBFNN algorithm for fractionalorder time delay systems", Soft Computing Vol.21, Issue.14, pp.4005-4018, 2016.

[28] Rahmat, M. F. A., Yazdani, A. M., Movahed, M. A., \& Mahmoudzadeh, S, "Temperature Control of a Continuous Stirred Tank Reactor by means of Two Different Intelligent Strategies", International Journal on smart sensing \& intelligent systems, Vol.4, Issue.2, pp.244-267, 2011.

[29] Glover. B, "Understanding flowers and flowering", (2nd ed.), Oxford University Press, 2014 .

[30] Chittka. L, Thomson.J. D, \&Waser. N. M. "Flower constancy, insect psychology, and plant evolution", Naturwissenschaften, Vol.86, pp.361-377, 1999.

[31] Waser, N. M. "Flower constancy: Definition, cause, and measurement", The American Naturalist, Vol.127, pp.593-603, 1986. 